\title{
Sable, moules, modèles et matrice
}

Le cycle plastique d'une fonderie marocaine

Sand, mould, pattern and matrix

\section{Baptiste Buob}

\section{(2) OpenEdition}

Journals

Édition électronique

URL : https://journals.openedition.org/tc/7242

DOI : $10.4000 /$ tc. 7242

ISSN : 1952-420X

Éditeur

Éditions de l'EHESS

\section{Édition imprimée}

Date de publication : 15 décembre 2013

Pagination : 122-143

ISBN : 978-2-7351-1654-6

ISSN : 0248-6016

Référence électronique

Baptiste Buob, «Sable, moules, modèles et matrice », Techniques \& Culture [En ligne], 61 | 2013, mis en ligne le 15 décembre 2016, consulté le 29 septembre 2022. URL : http://journals.openedition.org/tc/ 7242 ; DOI : https://doi.org/10.4000/tc.7242 


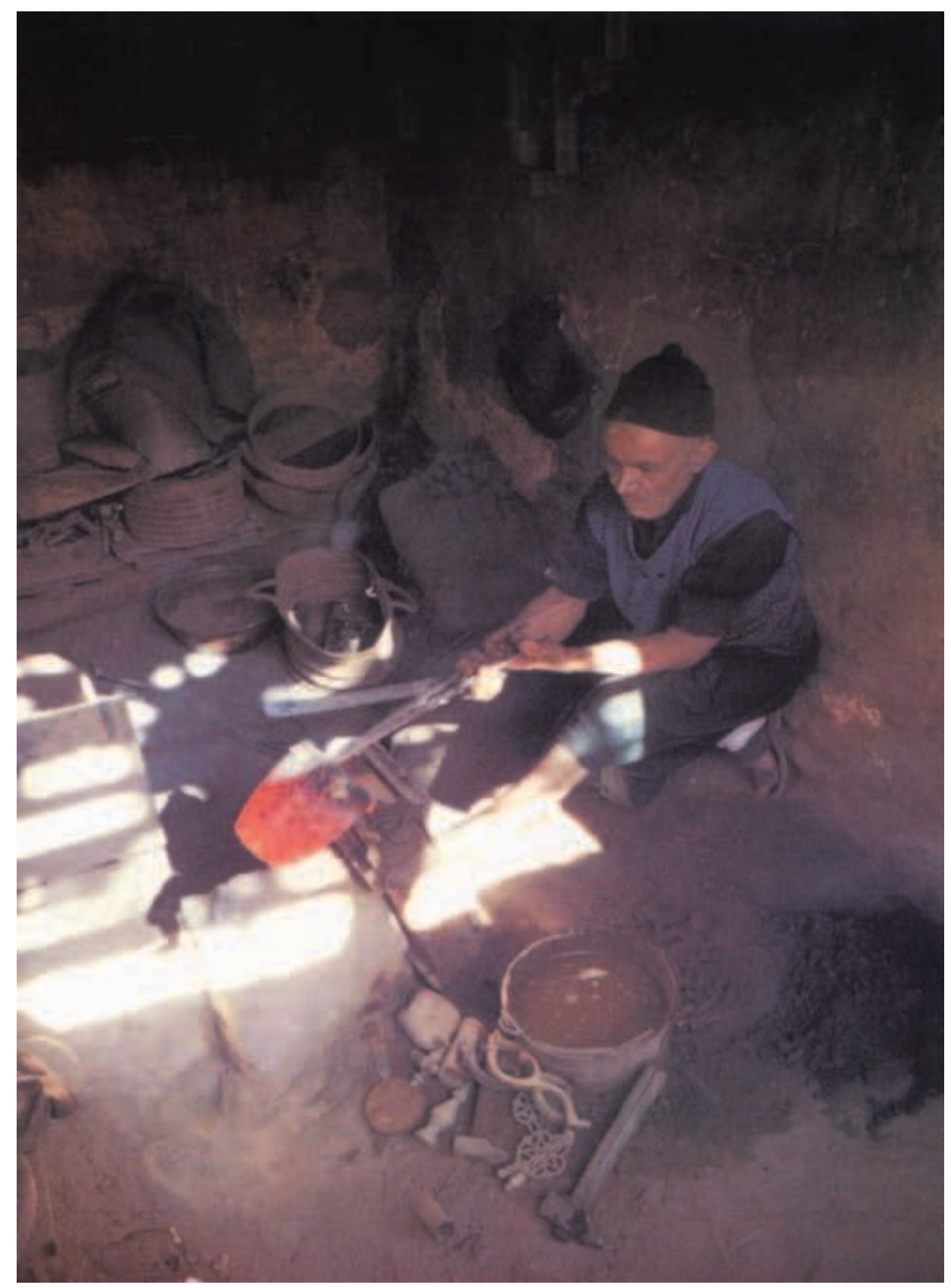




\section{SABLE, MOULES, MODÈLES ET MATRICE}

\section{Le cycle plastique d'une fonderie marocaine}

La «fonte au sable » est une technique de fabrication d'objets par coulage de métal en fusion dans des moules à usage unique empreints en creux de la forme d'un ou plusieurs modèles à reproduire. Récipients éphémères capables de recevoir et conserver l'empreinte d'un objet en trois dimensions et de résister au coulage de métal en fusion, les moules sont composés d'un «sable » tantôt qualifié de « naturel », lorsqu'il est issu de carrières, tantôt qualifié de «synthétique », lorsqu'il est produit par la réunion de silice, d'argile et d'adjuvants. Les guillemets s'imposent car les «sables de fonderie » avec lesquels sont formés les moules ne sont jamais « purs » mais toujours, dans des proportions variables, le produit d'un mélange ${ }^{1}$ - par exemple, de l'eau et un liant favorisant l'agglomération et le durcissement sont ajoutés au sable dit «naturel ». Sélectionné, purifié, nettoyé et mélangé, le sable de fonderie a été modifié par l'action de l'homme ; à l'instar de l'argile d'un briquetier (Simondon 2005 [1964] : 41) ou du sable d'un verrier (Magny 1869), les artisans n'utilisent généralement pas une matière existant à l'état brut, mais un matériau qui est le produit, à un degré ou à un autre, d'une transformation.

$\mathrm{Au}$ Maroc, deux techniques de fonte au sable coexistent. La première s'effectue avec du sable «naturel » de couleur ocre collecté dans le pays, la seconde avec du sable «synthétique » de couleur noire - une variante de la technique usuellement qualifiée de fonte au sable « à vert ». Cette dernière technique, notamment pratiquée à Marrakech pour l'obtention de mortiers, est certainement d'introduction récente ${ }^{2}$ tandis que celle effectuée avec du « sable jaune » est très ancienne même si, on le verra, son histoire est difficile à retracer ${ }^{3}$. C'est de cette seconde technique, et plus spécifiquement de la phase de confection des moules, dont il sera question.
La langue, comme c'est souvent le cas, a tendance à glisser du processus au résultat, du point de départ (le moule, ou forme négative) au point d'arrivée (le tirage, ou forme positive). Mais nous ne devons pas isoler les termes les uns des autres, faute de quoi c'est le système tout entier qui deviendra incompréhensible. Or l'empreinte est bien ce système: forme et contre-forme réunies en un même dispositif opératoire de morphogénèse. Didi-Huberman, 2008 : 53 
Les données mobilisées sont issues d'une enquête menée aux côtés d'artisans dinandiers de la médina de Fès dans le cadre de laquelle fut réalisé le film Fonderie, consacré à la description suivie du processus opératoire de la fonte au sable (Buob 2009b ; le film est également accessible à l'adresse http://vimeo.com/76040806) ; aussi, les deux premiers temps du présent texte, consacrés à l'histoire de la fonte au sable au Maghreb puis à la description de sa pratique actuelle, exploitent des données déjà en partie présentées antérieurement. Après avoir fait le constat de la rareté des données historiques concernant la technique de fonte au sable au Maghreb, malgré son ancienneté avérée, il s'agira de rendre intelligible le processus de fabrication filmé. Outre les difficultés cognitives propres à la compréhension des techniques de moulage, une telle description est rendue complexe par le fait que les actuels fondeurs de la médina de Fès, loin de l'image d'artisans exerçant seuls leur activité en vue de la confection de pièces uniques, fabriquent de grandes quantités de pièces coulées en utilisant un procédé favorisant la production sérielle.

Partant du cas spécifique de la fonderie filmée à Fès, la dernière partie propose une réflexion plus générale sur la notion de plasticité qui, une fois définie, m’a amené à reconsidérer la logique technique à l'ouvre lors du processus de fabrication des pièces de métal avec des moules en sable. D’abord influencé par l'approche praxéologique des techniques matérielles promue dans le domaine des recherches usant des outils audiovisuels (de France 1983), laquelle revient à considérer un continuum technique en privilégiant une approche de type fonctionnaliste des éléments qui composent le processus opératoire (objets, matières, outils, agents), j’ai été dérouté par l'observation de la fonte au sable, et ce pour au moins deux raisons : d'une part parce que l'objet recherché demeure longtemps virtuel, simplement présent « en creux »; d'autre part, parce que les composantes mobilisées dans le cadre de l'analyse praxéologique (matière/outils/objets) se révèlent très ambiguës - dans le sens où il semble particulièrement arbitraire et circonstanciel de ranger certaines composantes techniques dans telle ou telle catégorie. Corroborée par l'analyse sémantique d'un terme essentiel du technolecte local (qâleb), l'hypothèse ici proposée est que la particularité des activités de moulage tient au fait qu'elles associent deux corps « plastiques » (le « sable » et l'alliage métallique), des matériaux qui, en fonction de leur état au cours du processus, sont tantôt donneurs de forme, tantôt réceptacles de forme ; tantôt des « plastes », tantôt des « plasmes ». Penser le moulage à partir de telles notions, empruntées tant à la philosophie qu'à la science des matériaux, conduit à envisager les actes de transformation comme des boucles de métamorphoses successives.

Ainsi, tout en restituant des données spécifiques à la fonte au sable marocaine, l'objectif est de proposer un cadre d'analyse plus ample, susceptible d'éclairer la logique technique permettant d'obtenir des objets a priori aussi incommensurables qu'une sculpture d'Auguste Rodin, un étrier de laminoir en acier gardois, une fibule en argent algérienne, une marmite en aluminium sénégalaise, un brûle-parfum en bronze vietnamien ${ }^{4} \mathrm{ou}$, comme c'est le cas ici, une poignée de théière ou des pieds de plateau en laiton marocains.

\section{Traces historiques}

Si la fonte au sable se pratique depuis des millénaires de l'Europe à la Chine (LeroiGourhan 2000 [1943] : 200-201), des données précises concernant les processus, leur 
diversité et leurs évolutions au Maghreb sont rares. On sait aujourd'hui que l'Afrique du Nord a connu un âge du bronze vers la fin du $\mathrm{II}^{e}$ millénaire avant notre ère ${ }^{5}$ et que les objets « protohistoriques » d'alors étaient obtenus par martelage et moulage (Benfoughal 1997 : 29). Durant l'Antiquité, les Phéniciens, les Romains, les Vandales ou encore les Byzantins y ont sans aucun doute transformé des métaux. Si les Vandales introduisirent l'émail cloisonné (Camps-Fabrer 1970) et que l'antiquité fut une ère de fort dynamisme métallurgique (Gsell 1901, Arharbi 2003), les apports de ces diverses populations furent minimes auprès des populations autochtones (Benfoughal 1997). C'est surtout au moment de la conquête arabe que se renouvellent les pratiques métallurgiques locales ${ }^{6}$. Il existe d'ailleurs deux anciens moules réutilisables (apparemment en terre cuite gravée) utilisés pour fondre des bijoux dont les formes et les motifs confirment l'influence de l'Orient musulman sur certaines pratiques locales : le premier, trouvé à Volubilis, a pu être apporté dès l'époque proto-islamique à la fin du viII siècle ; le second, trouvé à Dchira, daterait du XI ${ }^{\mathrm{e}}$ ou xil ${ }^{\mathrm{e}}$ siècle (Rulhmann 1935).
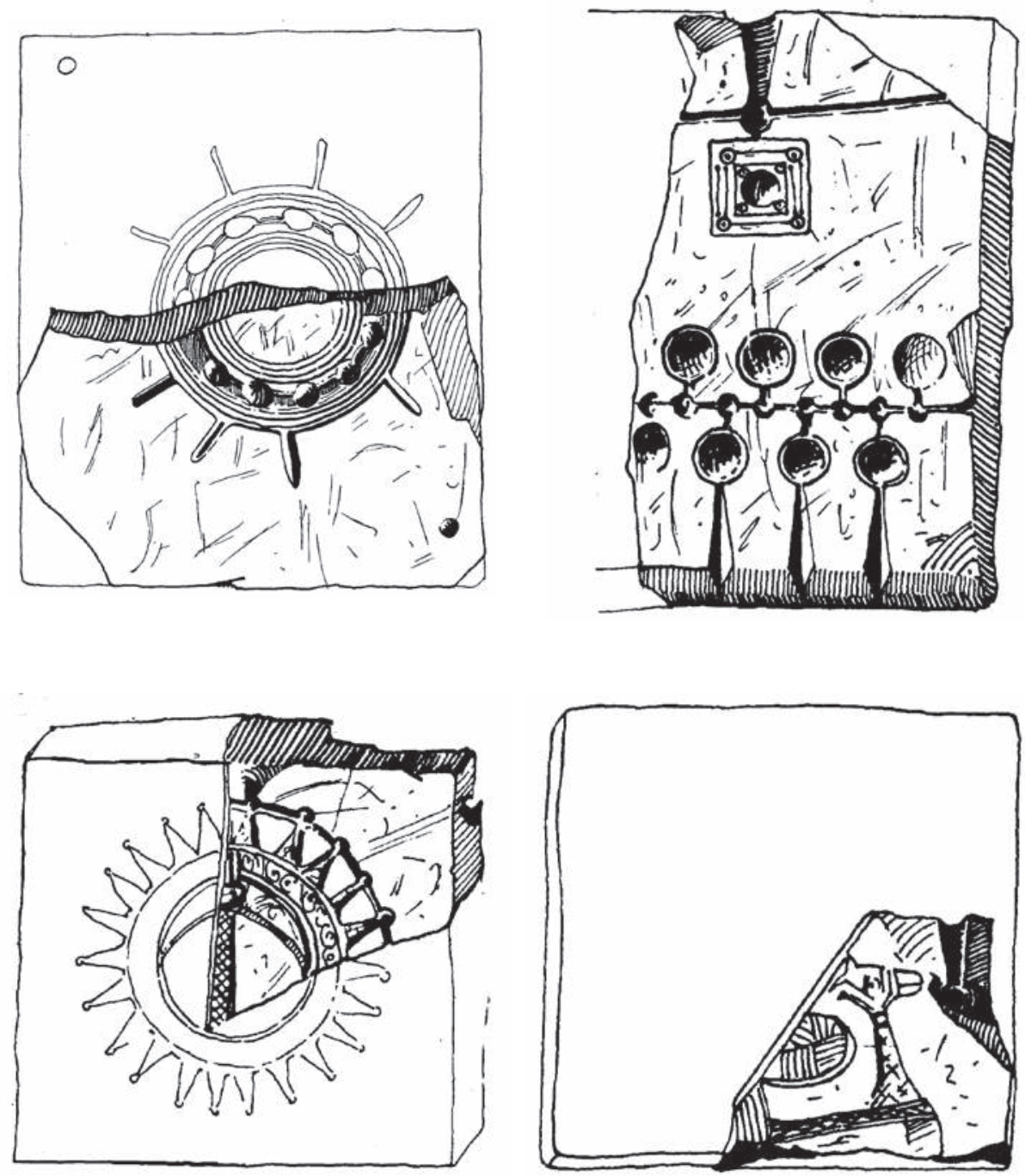

Les deux parties du moule en terre cuite de Volubilis (Rulhmann 1935 : 142-143)
Les deux parties du moule en terre cuite de Dchira (Rulhmann 1935 : 144-145) 


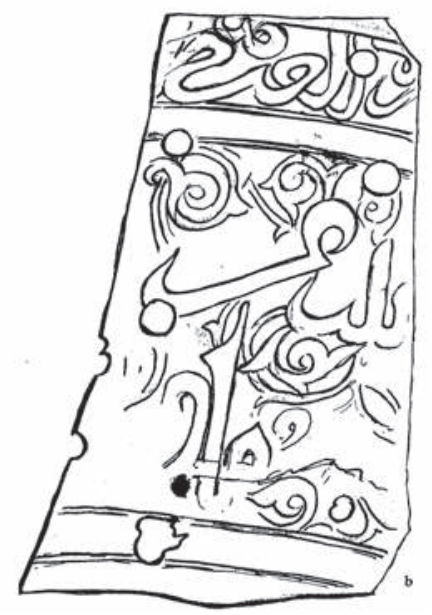

Fragment d'un plateau du xu ${ }^{e}$ ou du xille siècle retiré d'un creuset avant sa fonte intégrale (Delpy $1958:$ 167).

Lustre de la mosquée Qarawyine de Fès et cloche andalouse en bronze qui aurait été fondue pour sa confection (Paccard $1981: 457$ ).
Outre ces influences orientales, les juifs (et notamment ceux expulsés d'Andalousie par Isabelle la Catholique) furent des agents directs tant de la circulation des savoirs et des pratiques que du dynamisme économique dans le domaine métallurgique (El Koutbia 1992). D'ailleurs c'est essentiellement cette origine qui a marqué les actuels dinandiers de Fès, nombreux à affirmer que leurs activités étaient initialement effectuées par des artisans juifs ${ }^{7}$. Concernant la ville de Fès, nous savons qu'elle a compté une douzaine de fonderies de cuivre au xIII ${ }^{e}$ siècle (Ibn Abi Zar' 1990 [1326] : 48), qu'à partir du xvl e siècle, avec l'aide de Turcs, d'Anglais et de Moresques, ces fonderies s'orientent vers la production d'armes (Laborie \& Tag 1990 : 42) et que la fabrication de pièces moulées est presque totalement mise à mal par la concurrence des objets européens au début du xx siècle (Terrasse \& Hainaut 1925 : 159). Ainsi, malgré le rôle essentiel joué par la métallurgie et ses produits dans l'histoire du Maghreb occidental (Colin 1936, Jodin 1966, Rosenberger 1970), le chercheur ne dispose d'aucune donnée précise. Aussi on ne peut qu'émettre l'hypothèse que la fonte au sable a pu permettre aux orfèvres maghrébins de fabriquer de nombreux bijoux en cuivre (Grébénart 1994) et aux dinandiers de confectionner des objets comme de massives poignées de porte en bronze et d'autres lustres caractéristiques de l'artisanat marocain (Cambazard-Amahan 1989).

Les données concernant spécifiquement la fonte au sable sont très rares et il est peu probable que ces connaissances historiques s'accroissent. Il est, en effet, difficile d'identifier les objets obtenus grâce à cette technique : les traces attestant cette pratique sont visibles sur les objets tant qu'ils ne sont pas finis - une fois les pièces terminées, les indices directement visibles ont généralement disparu, même si dans certains cas une rugosité et des « piqûres » caractéristiques de la fonte au sable peuvent apparaître (Gaube du Gers 1993 : 36). Il faudrait pouvoir étudier des pièces non finies, mais celles-ci ont sans aucun doute été recyclées ; pratiqué de façon systématique pour les pièces devenues défectueuses et couramment pour les objets hors d'usage, le recyclage consiste à refondre des pièces pour en obtenir de nouvelles. Le plus ancien fragment de plateau connu dans l'aire géographique du Maroc actuel, datant probablement du XII ou du XIII siècle, aurait d'ailleurs été retiré d'un creuset avant sa fonte intégrale (Delpy 1958 : 167). Le recyclage était aussi pratiqué à plus grande échelle lorsqu'il devenait nécessaire de fondre bijoux et vaisselles métalliques afin de frapper de la nouvelle monnaie, notamment lors des périodes de crise et d'épidémie (El Koutbia 1992 : 20-21).

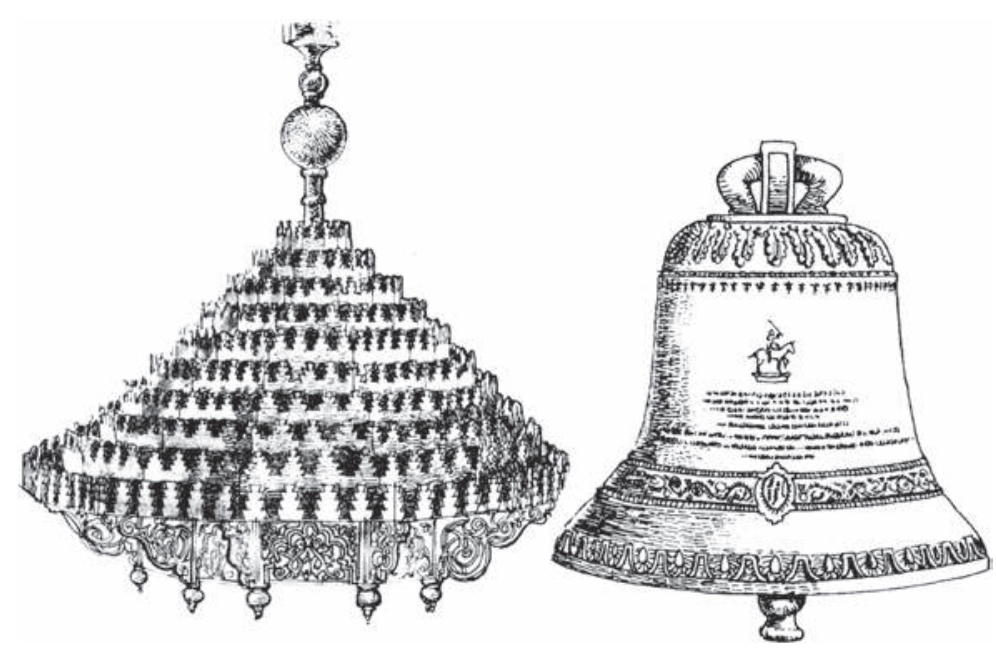


Il est par ailleurs quasi inespéré de découvrir d'anciens moules en sable puisque leur avantage est justement lié à leur impermanence et qu'ils sont toujours détruits lors de la phase de décochage ${ }^{8}$. De façon générale, il est donc très malaisé de certifier à partir d'un artefact que la fonte au sable a été pratiquée (Mordant \& Pernot 1998). Les données historiques sont insuffisantes pour comprendre les façons de faire et a fortiori leurs évolutions. La fonte au sable marocaine n'a pas laissé de trace, le sable est redevenu pulvérulent, le métal a été refondu.

Il existe toutefois quelques données plus récentes sur la fonte au sable au Maghreb. Concernant la dinanderie, une douzaine de photographies légendées permettent de saisir quelques bribes de ce qui pourrait relever d'une ancienne méthode de fonte au sable à Fès (Paccard 1981), méthode proche de celle employée pour le moulage de bijoux en Algérie (bracelets, fibules, etc.) ayant fait l'objet de quelques descriptions relativement récentes (Riché 1961, Camps-Fabrer 1970, Benfoughal 1992 et 1997).

« Avec [un mélange de sable jaune et d'huile d'olive] le bijoutier remplit séparément les deux châssis du moule, posés sur une planche en bois, après quoi il le tasse, l'aplanit et le saupoudre de charbon pour éviter que les deux parties du moule, une fois réunies, ne se collent entre elles. Vient ensuite le façonnage des empreintes. Pour cela, il place les modèles à reproduire sur la face interne de l'un des châssis, de part et d'autre d'une petite barre dont l'empreinte, dans le prolongement du goulot, constituera la conduite principale ou maître-jet, destinée à assurer le passage du métal liquéfié. Il réunit ensuite les deux châssis du moule qu'il martèle à petits coups de maillet sur leur face externe pour bien imprimer les modèles, puis il ouvre le moule et en retire les modèles. Avec une tige métallique il trace alors les nervures entre le conduit principal et les empreintes des modèles, afin de les relier à celui-ci. Il referme le moule et le fixe entre les deux planches du serre-joint, en le plaçant en position verticale. Le creuset tenu avec une pince, il ne reste plus qu'à verser le métal en fusion dans le goulot d'où il se répartit dans toutes les cavités. Après un quart d'heure, il procède au démoulage. Suivent enfin l'élimination des bavures et la finition des bijoux ainsi obtenus (limage, nettoyage et polissage). » (Benfoughal 1997: 212-213)
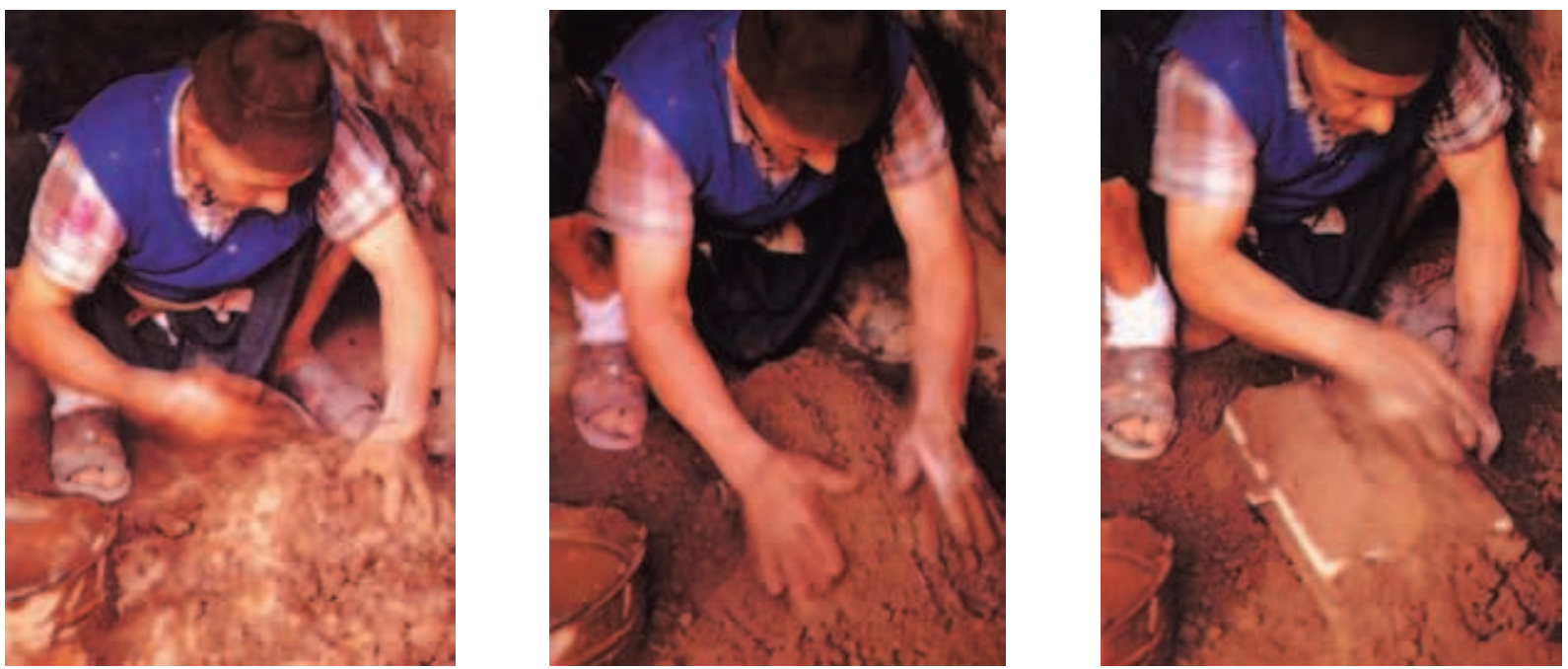

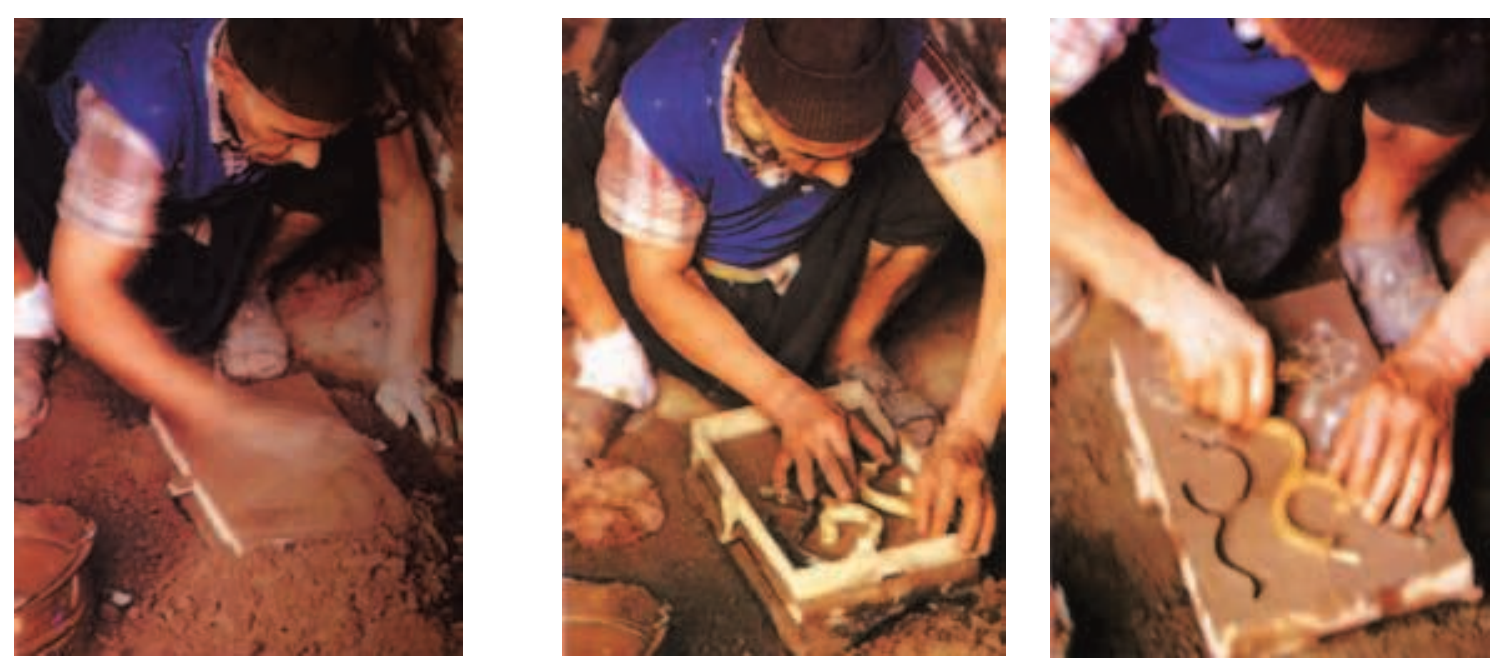

La fonte au sable à Fès dans les années 1980 (Paccard $1981: 460-463)$
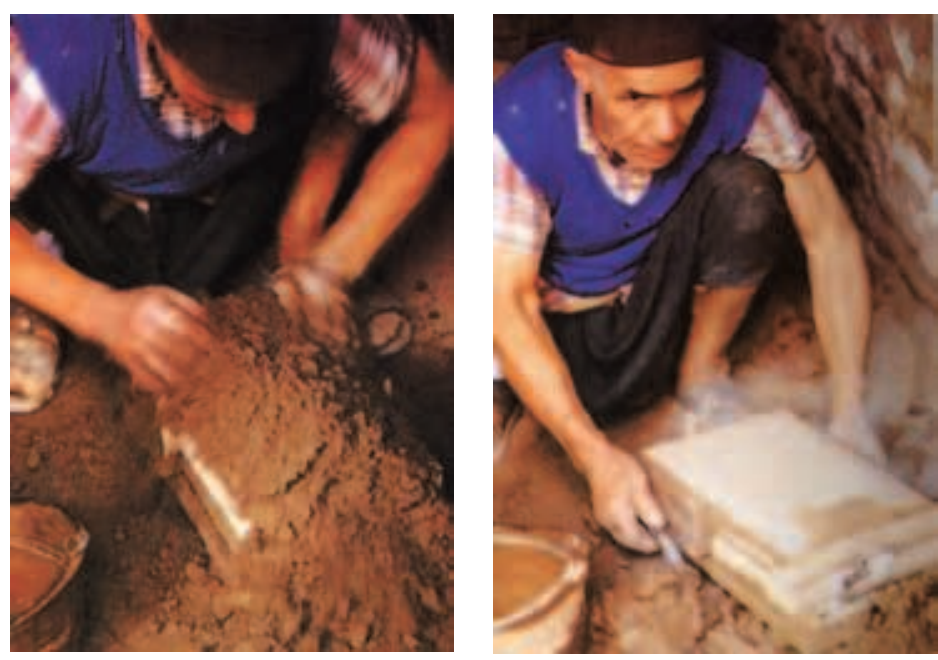

La logique de ces techniques décrites et photographiée est proche de celle que j’ai filmée à Fès (Buob 2009b). En revanche, le contexte de fabrication est très différent : tandis que sur les photographies et dans les descriptions écrites les artisans travaillent seuls à l'élaboration de moules uniques, les dinandiers filmés confectionnent des séries de moules identiques pour produire des pièces en grande quantité. Les dinandiers fassis n'utilisent pas une technique de moulage classique mais recourent à une technique de fonte au sable dite " par empilement $»^{9}$.

Cette méthode s'est probablement développée dans l'entre-deux-guerres, lorsque la dinanderie s'est mise à produire des ustensiles pour la consommation du thé. En effet, c'est durant cette période qu'on voit apparaître des pièces de garniture ajoutées aux plateaux qui n'étaient jusqu'alors composés que d'un seul élément : « La pose de pieds surajoutés au plateau traditionnel » (Berque 1962 [1939] : 191) était sans doute effectuée avec des éléments fabriqués dans les fonderies de la Médina. C'est également à cette époque que les fondeurs entreprennent de couler des objets nouveaux 
comme des théières en étain (ibid. : 315). Après la Seconde Guerre mondiale, avec l'implantation progressive du tour à repousser électrique dans la Médina (Houssel 1966 : 67), les théières peuvent être fabriquées plus simplement que par le procédé de fonte de pièces en étain, lequel tombe en désuétude. Quelques artisans vont continuer à fabriquer seuls des pièces comme des poignées de portes, tandis que certaines fonderies s'orientent vers la confection exclusive de petites pièces de garniture (poignées, pieds, éléments de décoration, etc.) qui, achetées par d'autres dinandiers, seront assemblées à divers objets. Ce changement dans l'organisation du métier a été rendu possible par la place croissante cédée progressivement à la mécanisation, à la division des tâches et à la parcellisation du savoir transformant progressivement les artisans en ouvriers (Buob 2009a). Si la logique de la fonte au sable actuelle est proche de celle suivie par un bijoutier ou un dinandier exerçant seul son activité, elle nécessite cependant des moyens techniques, humains et financiers tout autres.

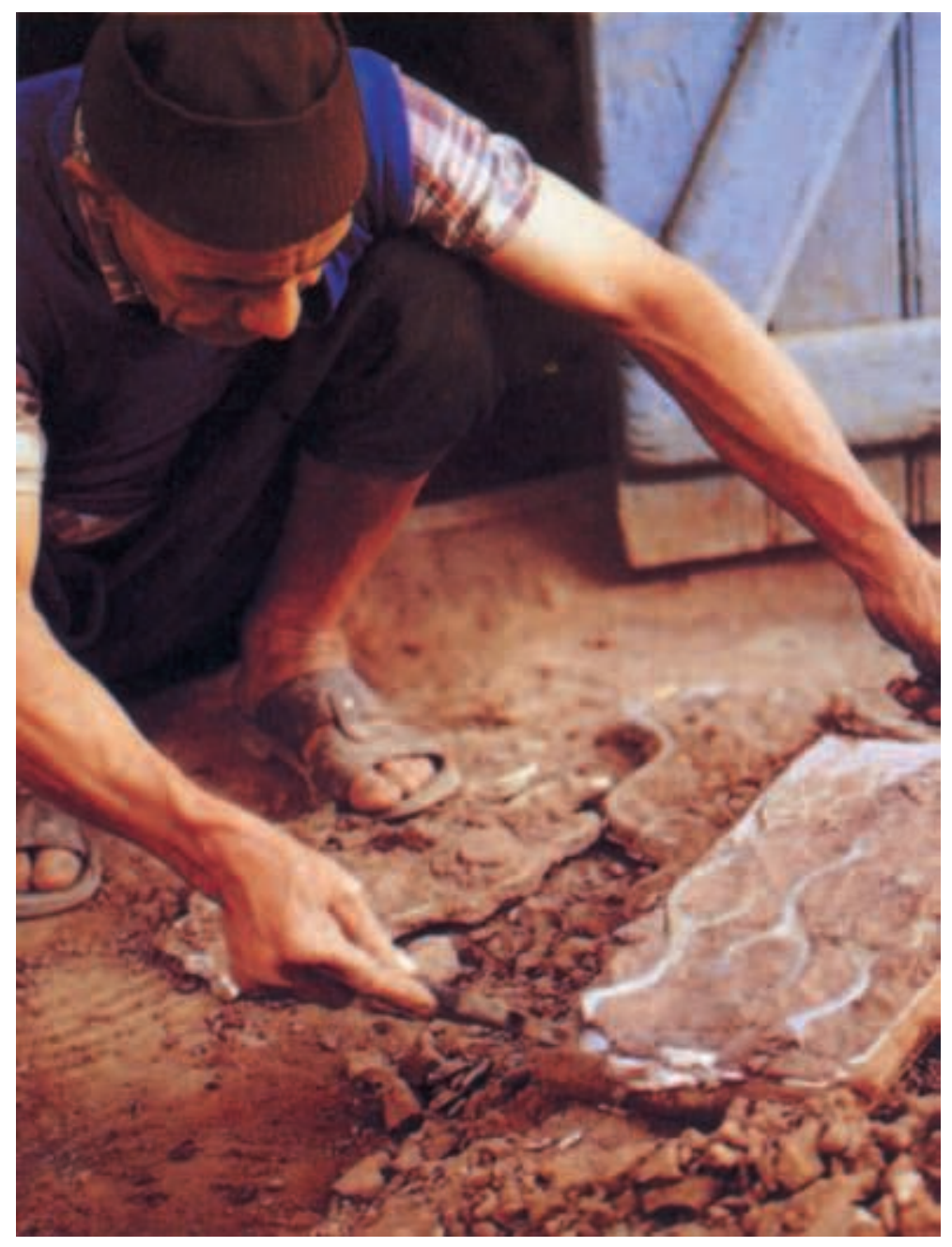




\section{Technique du moulage}

Le processus de fabrication de pièces coulées observé à Fès peut être divisé en quatre phases : la préparation de moules, la fonte d'un alliage dans un creuset, le coulage de l'alliage en fusion dans les moules et les finitions. Sept agents s'occupent des opérations allant du moulage jusqu'au décochage, et une demi-douzaine d'autres s'occupent des multiples opérations de finition. Ainsi, pour les opérations qui précèdent les finitions : deux enfants ont pour attribution d'assister les mouleurs et de pratiquer le décochage ; deux ouvriers nettoient et transportent le sable de fonderie, s'occupent également de la cuisson des moules, enserrent les moules préalablement au coulage puis les desserrent une fois le coulage terminé ; un autre ouvrier est en charge de la préparation du four et de la fusion de l'alliage dans le creuset ; enfin deux artisans confirmés s'occupent de la confection des matrices, du moulage et du coulage.

Dans le contexte des fonderies de Fès, organisées autour de la reproduction en série de pièces simples (de petites dimensions et sans trop de creux ou de bosses), le moulage occupe une place plus importante que la transformation du métal, tant en terme de temps de travail que de représentations. Le lexique utilisé à Fès suggère d'ailleurs l'importance accordée au coulage et au moulage. Utilisé pour désigner une fonderie, le terme frigh ne désigne pas tant le lieu où se pratique l'art de fondre et de purifier les métaux, que l'espace où l'on procède au coulage de métal dans des moules. L'activité la plus valorisée parmi les fondeurs consiste d'ailleurs, selon eux, à confectionner les moules, à les « marquer » (rshem). C'est principalement de ces activités de moulage dont il sera ici question ${ }^{10}$.

La phase de moulage consiste à confectionner plusieurs centaines de moules à partir de « sable jaune ». Les moules utilisés lors de la fonte au sable ne sont pas confectionnés à partir de n'importe quel sable et l'usage même de ce terme, nous l'avons vu en introduction, doit être sujet à caution. Dans le contexte français la distinction entre sable et terre relève de l'arbitraire (Lebon 2012), aussi n'est-il pas surprenant qu'André Paccard, dans la seule étude consacrée à cette technique à Fès, emploie le mot englobant « terre » pour désigner le matériau utilisé pour former les moules, alors que les artisans que j'ai rencontrés préfèrent le terme remla [sable]. Quoi qu'il en soit, la nature exacte de ce matériau n'est pas connue : les artisans de Fès ignorent tant le processus de formation que la composition ${ }^{11}$ de ce sable qui arrive après avoir été transformé et conditionné ${ }^{12}$ aux environs de la ville de Sefrou située à une trentaine de kilomètres.

Au départ d'un nouveau cycle, le responsable de l'atelier sélectionne les pièces à faire fabriquer en fonction de l'état de ses stocks et/ou de commandes spécifiques. Ces modèles sont en l'occurrence des pièces de garniture en laiton. Comme certains de ces modèles sont symétriques (les poignées de théières par exemple), mais que d'autres possèdent deux côtés et ne sont pas réguliers (les pieds et les becs de théières), les dinandiers distinguent le revers de l'avers des modèles. Cette opposition permet de différencier les deux parties du moule qui seront finalement obtenues. Le dos d'un moule est le châssis où est imprimé en négatif le revers des pièces, la face d'un moule est le châssis où est imprimé en négatif leur avers décoré.

Afin de produire une série de moules semblables, les artisans commencent par confectionner une matrice ${ }^{13}$ à partir des modèles. Comme on le verra, la matrice servira elle-même de modèle pour obtenir des dos de moules, lesquels seront à leur tour utilisés pour obtenir les faces de moules correspondantes. Pour l'obtenir, le mouleur dispose les modèles sur un 
châssis préalablement rempli de sable, tassé à la main puis à l'aide d'un maillet, et arasé avec une réglette en fer. Il doit laisser suffisamment d'espace entre les modèles afin de creuser par la suite les jets (réseau de conduits par lesquels l'alliage pourra s'écouler) et des encoches grâce auxquelles les deux châssis du moule, une fois réunis, seront parfaitement imbriqués.

Le châssis est doté d'une entaille, un « entonnoir de coulée », par où sera versé l'alliage. Sous l'entaille est disposé le "guide maître jet », qui servira de base pour creuser le réseau de conduits par lequel s'écoulera l'alliage en fusion dans le moule. Le mouleur positionne les pièces asymétriques afin que la partie décorée des pièces soit visible. Une fois les modèles disposés, il marque leur empreinte dans le châssis rempli de sable. Il commence par « imprimer $»^{14}$ les modèles dans le sable compacté à l'aide d'un percuteur métallique. Il ôte ensuite les pièces dont le contour est dès lors imprimé dans le sable. Puis, avec une petite lame, le mouleur creuse la surface du sable en suivant les empreintes laissées par chacun des modèles afin de pouvoir, ensuite, les enfoncer à la moitié de leur hauteur dans le sable du châssis.

Lélément obtenu ne correspond pas à la pièce recherchée, il s'agit en fait du positif à partir duquel sera obtenue la matrice définitive. Afin d'obtenir un négatif de cet élément, le mouleur répand généreusement de la poudre de charbon sur le premier châssis doté des modèles. Cette opération effectuée, il remplit de sable un second châssis, disposé avec précision sur le premier, et le « serre $»^{15}$, d'abord à la main, puis à l'aide d'un maillet. Le sable contenu dans le second châssis prend alors l'empreinte de l'avers des modèles imprimés dans le sable du premier châssis.

Ensuite, il retourne les deux châssis et ôte le premier alors placé sur le dessus de l'ensemble. Le châssis du dessous est la matrice recherchée : un châssis rempli de sable sur lequel sont imprimés les modèles en laissant apparaître en surface leur revers non décoré. Le mouleur parfait cette matrice en réparant les imperfections dues au démoulage. Lors de cette opération de « recharge jusqu'au plan de joint », le mouleur corrige à la main les imperfections de la matrice en ajoutant du sable là où il en manque et en égalisant la surface. Enfin, il creuse les encoches sur le pourtour du châssis qui permettront aux deux parties du moule obtenues à partir de la matrice de parfaitement se juxtaposer. Les retouches effectuées et les encoches creusées, il asperge généreusement la matrice avec de l'eau, la saupoudre de charbon finement broyé et la laisse reposer jusqu'au lendemain.

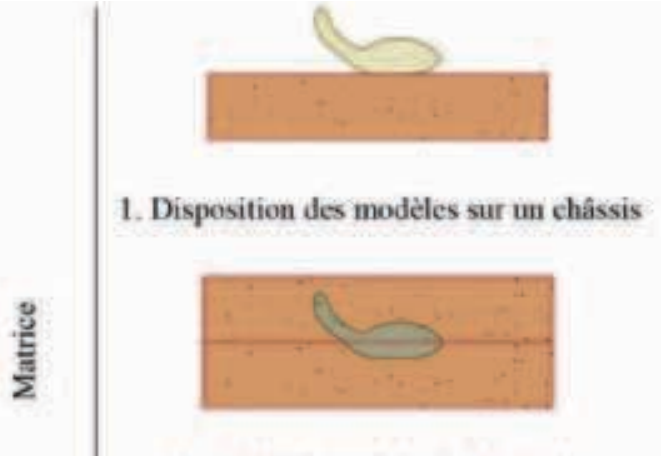

3. Dressage d'un second châssis

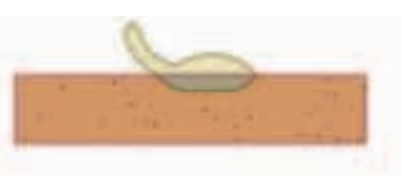

2. Impression des modèles

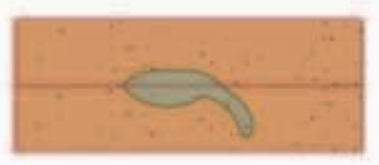

4. Retaumement des deux chấssis

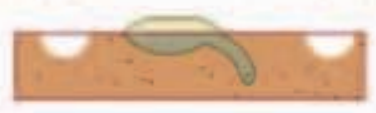

5. Séparation, réparation de la couche et creusage des clés

Confection de la matrice 
La matrice, rappelons-le, sert de modèle pour obtenir les dos de moules (les demi-moules empreints du revers des modèles). Pour ce faire, le mouleur dispose un châssis sur la matrice dans laquelle le revers des modèles est apparent. Il ajoute une fine couche de poudre de charbon puis remplit le châssis avec du sable, dressé à la main, bourré vigoureusement puis damé à l'aide d'un maillet. Après avoir effectué ces opérations de dressage, le mouleur arase le dessus du châssis avec une réglette afin d'obtenir une surface parfaitement plane. Il sépare ensuite délicatement le châssis de la matrice, opération facilitée par le charbon préalablement saupoudré. Le dos de moule terminé est rangé contre une paroi à côté du dispositif. Le dinandier reproduit ensuite ce groupe d'opérations pour chacun des moules qu'il désire obtenir, environ trois cents en deux jours (soit un total de six cents moules pour les deux mouleurs).

Confection d'un demi-moule dos

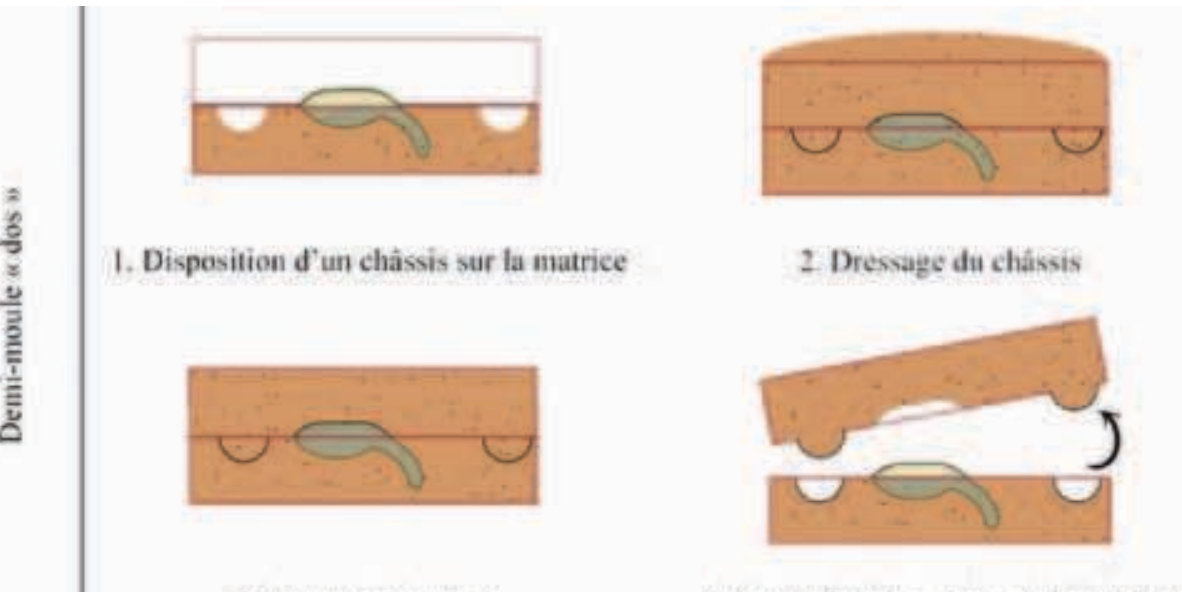

3. Arasage du châssis
4. Separation du $*$ dos $n$ de la matrice

Ce n'est qu'une fois qu'il a confectionné l'ensemble des dos de moules à partir de la matrice, que l'artisan entreprend d'effectuer les faces des moules. Il doit préalablement détruire la matrice afin de récupérer les modèles qu'il dispose ensuite dans les empreintes marquées sur un dos préalablement confectionné. Il effectue alors les mêmes opérations que pour la confection des dos de moules : il pose un nouveau châssis, saupoudre du charbon pulvérulent puis du sable qu'il dresse (à la main, avec ses poings et avec un maillet) et enfin arase le sable excédentaire. Une fois le sable du second châssis dressé et arasé, le mouleur retourne l'ensemble et désolidarise les deux châssis. Il retire ensuite les modèles de la face et répare la couche dégradée en ajoutant du sable par endroits. Afin de gagner du temps, un enfant dispose les modèles dans un autre dos à partir duquel sera obtenue la face complémentaire. Les dernières opérations de la confection d'un moule consistent à évaser l'entonnoir de coulée et à tracer les jets afin de permettre à l'alliage de s'écouler dans chacune des empreintes des modèles. Ces opérations effectuées, le dos et la face du moule sont réunis pour former un moule définitif. 


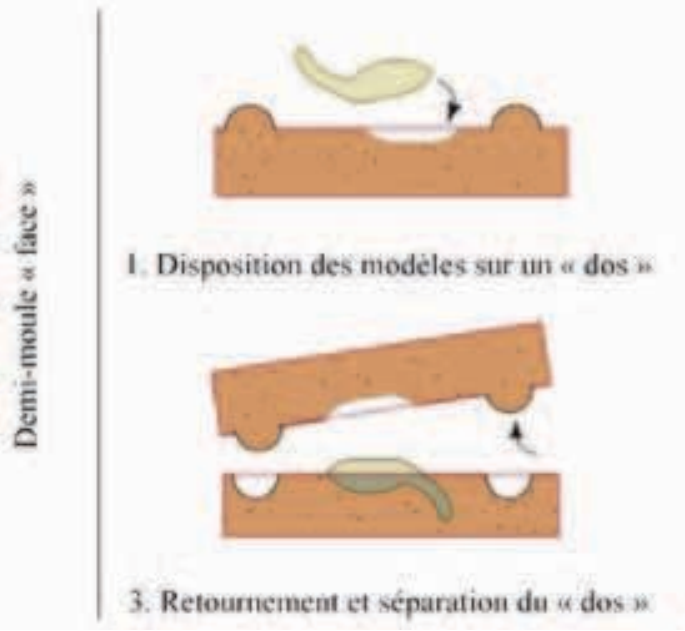

3. Retoumement et séparation du a dos $n$

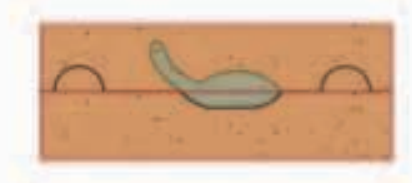

2. Dressage d'un chássis sur le $a$ dos w

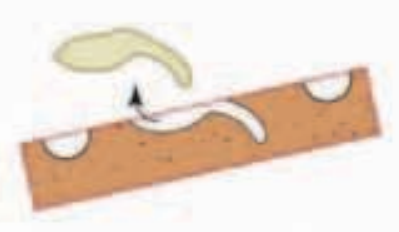

4. Démoulage et réparation de la couche

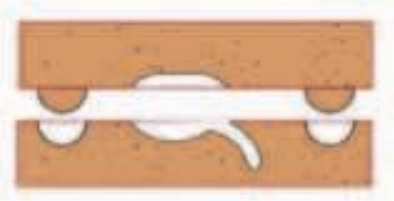

5. La $\alpha$ face w et le $\alpha$ dos $n$ réunis forment un moule
Le moule terminé

Parallèlement au moulage, d'autres artisans, qui s'occupaient de transporter le sable de fonderie jusqu'à l'espace de moulage, prennent en charge la « cuisson » des moules terminés. Les enfants travaillant aux côtés des deux artisans mouleurs emportent au fur et à mesure les moules terminés à proximité du lieu où ils seront cuits. Les deux parties de chacun des moules sont séparées et mises en contact avec le feu. Les agents font bien attention à disposer les deux parties de chacun des moules de façon à ne pas les mélanger par la suite (bien que réalisé à partir de la même matrice, chaque moule, dont face et dos ne sont pas interchangeables, est unique). Une fois «cuits» au contact des flammes du bois d'eucalyptus, les moules sont rangés en différents espaces de la fonderie en attendant la phase de coulage. Il s'agira enfin de réunir les éléments de chaque moule qui avaient été séparés et rangés à l'horizontale après leur cuisson. Ces opérations consistent principalement à souffler sur la face et le dos de chacun des moules afin d'enlever les éventuels dépôts, à les réunir et à les empiler les uns sur les autres. Cet ensemble d'opérations, en apparence simple à effectuer, est pris en charge par les maîtres artisans, car leur regard d'expert leur permet de vérifier d'un coup d'œil rapide que les deux parties correspondent bien à un même moule et qu'elles n'ont pas d'imperfections. Les enfants transportent progressivement les moules réunis dans l'espace de coulage, où d'autres les enserrent par piles de vingt dans un étau puis les couchent à l'horizontale avec les entonnoirs de coulée sur le dessus. Les moules sont alors prêts à recevoir l'alliage en fusion. 
Pendant que les deux dinandiers en charge du moulage procèdent au coulage de l'alliage dans les moules, d'autres desserrent la pile de moules déjà coulée, puis en préparent une nouvelle, tandis que les enfants procèdent au décochage. Au terme de toutes ces opérations, l'ensemble du métal ayant été versé dans les moules et le décochage effectué, un nouveau cycle de moulage peut débuter. Celui-ci commence par le tamisage du sable afin de l'homogénéiser en retirant les déchets de métal issus du précédent coulage qui seront réintroduits dans le suivant. Pour que le sable soit réutilisé il faut ensuite le mouiller pour assurer la liaison entre les grains et favoriser leur agglomération et ajouter éventuellement de la silice sous forme de «sable neuf ».

\section{Cyclicité plastique}

La description qui précède est assez compliquée et difficilement intelligible pour un lecteur non instruit dans les techniques de moulage. En effet, selon une lecture classique du processus, il faut considérer les pièces finalement coulées comme étant les positifs des deux parties de moules dont l'une (la face) est le négatif de l'autre (le dos) qui est lui-même le négatif d'un positif (la matrice) qui est elle-même le négatif d'un positif (le négatif de la matrice) qui est lui-même un positif obtenu par le serrage de sable entre les limites internes d'un châssis et les limites externes des modèles. Outre que ce type de description est malaisé à suivre, peut-on vraiment considérer la matrice, par exemple, comme un négatif? Si elle est le négatif d'un objet antérieur quand elle prend forme, elle constitue cependant le positif de l'objet postérieur quand elle lui donne forme. Comment dès lors considérer la matrice, le négatif de la matrice et plus généralement les moules au cours des différents groupes d'opérations? Faut-il les considérer comme des objets de l'action ou des outils? En fait leur statut diffère selon le moment où on les considère, le point de vue d'où on les observe : considérés comme des outils, ils sont les positifs contre lesquels le sable prend sa forme ; considérés comme des objets de l'action, ils sont des négatifs en train de prendre forme.

Le livre d'André Paccard fait figurer un terme vernaculaire qui permet d'envisager une voie pour mieux comprendre cette ambiguité : dans la légende des photographies 5 et 6 , le terme qâleb est utilisé pour désigner les « modèles » à reproduire ${ }^{16}$, en l'occurrence des marteaux de portes. Le terme est alors employé dans le même sens que celui des actuels mouleurs fassis. En revanche, pour Henriette Camps-Fabrer, le terme désigne un « moule» (2013 [1991] : § 4). Si dans son Dictionnaire des bijoux de l'Afrique du Nord, Paul Eudel traduit également le terme qâleb par « moule » (1906: 175), il semble pourtant, au vu des illustrations qu'il y fait figurer, qu'il s'agisse bien de modèles.

Le terme qâleb (pl. qouâleb) se traduit aussi bien par «modèle » que «moule, matrice », « forme », «format » ou «étalon » (Beaussier \& Ben Cheneb 2006 [1958] : 823). Le terme désigne à la fois ce qui permet de donner une forme au sable (modèle) et le sable ayant épousé cette forme (moule). Alfred-Louis de Prémare confirme cette double acception : d'une part, celle de «moule » et même de « moule creux (où l'on verse une substance pour qu'elle en garde la forme) » ainsi que, plus spécifiquement, de « moule où l'on coule du métal en fusion »; d'autre part, celle de « forme, patron, modèle, gabarit ${ }^{[17]} »(1998$ : 460). 

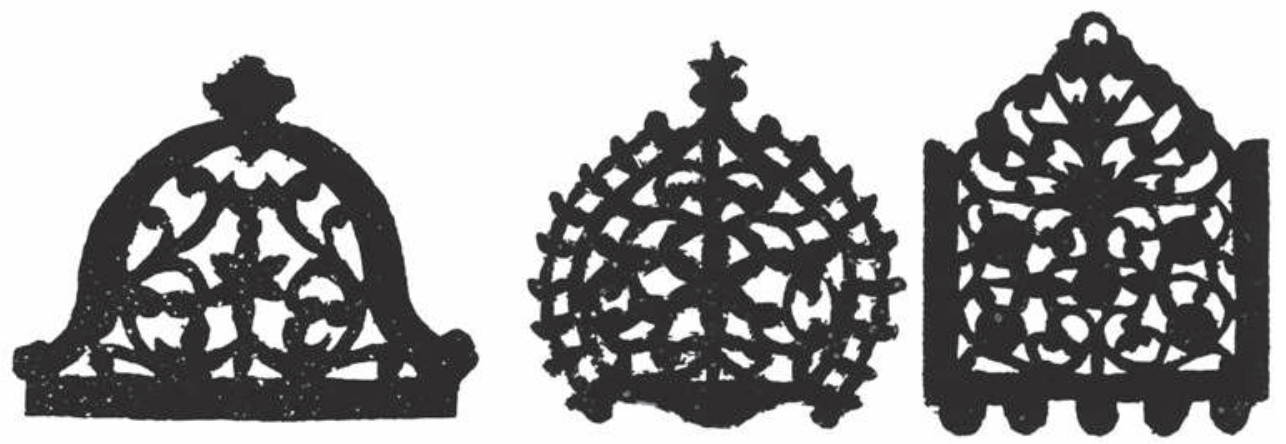

Qâleb, pl. Qouâleb - Nom du moule dont se servent les orfèvres arabes pour couler les bijoux (Eudel 1906 : 175 et 176 ; illustrations et légende)

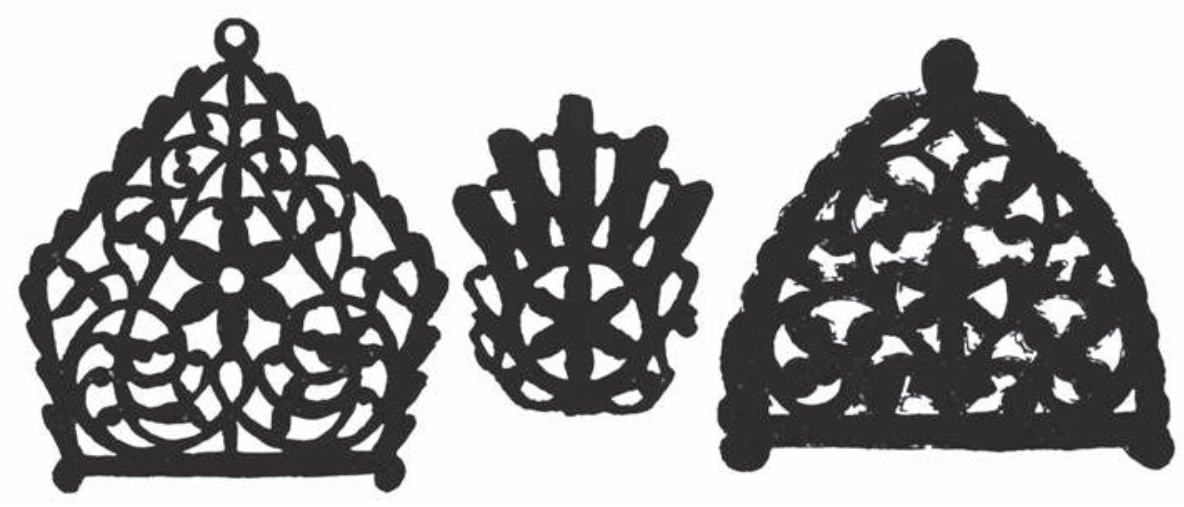

La racine QLB dont est issu le terme qâleb est elle-même ambiguë : si elle désigne le « cœur, centre, partie qui occupe le centre », son champ sémantique évoque également le fait de «tourner, mettre dans un autre sens, retourner » ou « renverser, mettre sens dessus dessous » (Beaussier \& Ben Cheneb 2006 [1958] : 822-823). Le terme qâleb luimême se renverse, se retourne selon le contexte et peut désigner à la fois un modèle et sa forme « inversée » (en l'occurrence le résultat de l'impression en négatif d'un modèle). Le même mot peut désigner ce qui permet de donner une forme, comme l'objet ainsi obtenu. Il serait à mon sens erroné de se contenter d'y voir un trope jouant sur le propre et le figuré, une simple métonymie (le tout pour la partie ; le contenu pour le contenant) ou synecdoque (le contenant pour le contenu; la partie pour le tout). Variant selon les contextes, selon les étapes de la fabrication, qâleb est un terme ambigu, amphibolique, qui contient en lui-même la possibilité d'un changement de catégorie. Selon le contexte technique, le qâleb sera un modèle ou un moule, un outil de la transformation ou l'objet de cette transformation, un positif ou un négatif. Le qâleb relève successivement ou alternativement de différentes catégories selon son état au cours de la fabrication ou la perspective depuis laquelle on l'observe. En un sens, le terme qâleb inclut l'idée même de transformation par contact.

Le sable, comme le métal, est porteur d'un potentiel de transformation et des ressources de sa propre évolution. Ce dynamisme ontologique est l'expression d’un caractère éminemment plastique. Selon André Leroi-Gourhan, les matières plastiques sont des « solides » qui ont le « caractère commun de pouvoir passer d'un état presque fluide à un état solide ou consolidé » et ils révèlent ce caractère plastique «si on leur impose une consistance qui permette de les 
modeler puis un traitement qui les fixe » (2000 [1943] : 206 et 207). Il distingue alors deux catégories : d'une part, les « semi-plastiques » qui acquièrent une malléabilité par échauffement (métaux) ; et d'autre part, les « plastiques » qui, malléables à l'état de traitement, peuvent acquérir une fixité par cuisson ou dessiccation (argiles, sables, etc.) (ibid. : 19). Un plastique se définirait donc comme une matière que l'on peut modeler et que l'on peut fixer de façon stable grâce à un changement d'état (fluide->solide / solide->fluide) ; il est ce malléable auquel on peut appliquer une forme pérenne. Mais à cette acception univoque de la nature plastique manque son autre versant à la fois complémentaire et opposé. Comme le propose la philosophe Catherine Malabou, une matière plastique n'est pas simplement malléable : elle est « ce qui est susceptible de recevoir comme de donner la forme » (2005: 33). Dans la continuité des réflexions que la philosophe mène sur le thème de la plasticité, Éric Combet (2009: 2) revient sur cette «nature contradictoire » :

« [...] la plasticité est tantôt la chose formée (ce qui se dit plasma en grec), et tantôt l'activité formatrice (que les Grecs appelaient plastikê, de plassein, modeler). Il faut donc distinguer dans la plasticité la passivité d'une plasmatique (la simple malléabilité), et l'activité d'un plastique, et par suite les êtres formés, qu’on appellera plasmes, et les êtres formants, qu'on appellera plastes. »

À condition de renverser l'idée de passivité du plasme et d'activité du plaste ${ }^{18}$, cette terminologie permet de considérer différemment la logique du moulage car elle réunit dans un même schéma les deux termes de la logique de la prise/dation de forme. Le processus de moulage de la fonte au sable apparaît alors comme un ensemble de métamorphoses : la transformation du sable est le produit de la rencontre de plasmes et de plastes rendue possible par le potentiel énergétique de l'artisan. Réunis par les gestes de pression de l'artisan, le sable non serré et le métal en fusion sont plasmes, les châssis et les qouâleb (modèles et moules) sont plastes.

\section{Plasmes et plastes de la fonte au sable}

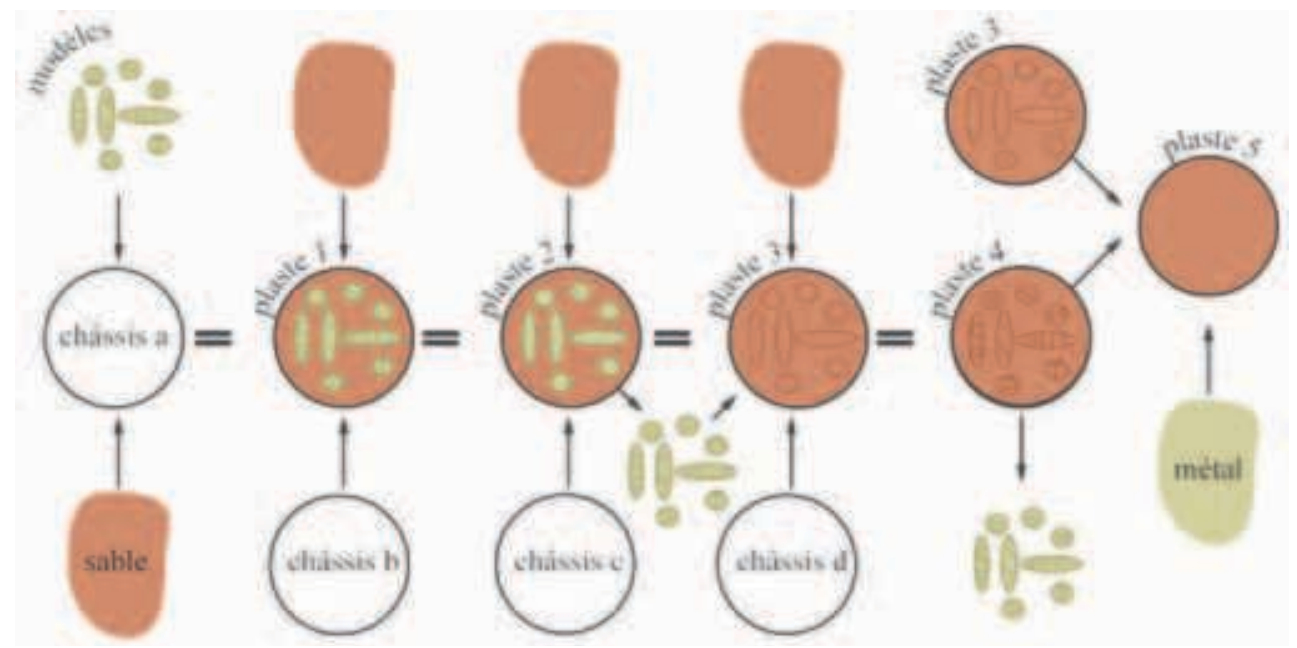

Les composantes du processus ont des fonctions variables, passant successivement d'un « état de traitement » à un « état d'emploi », selon les catégories établies par André 
Leroi-Gourhan, (2000 [1943] : 207). L'objet de l'action artisane change continuellement lors de la fonte au sable et la question de la « relativité des catégories » apparaît fondamentale pour en comprendre la logique ${ }^{19}$; l'ambiguité du terme qâleb venant en confirmer la pertinence. Cependant la plasticité, comme le souligne Catherine Malabou (2000 : 8), est un schéma à trois termes, à deux temps : «la réception et la prise de forme d'un côté (est dit "plastique" ce qui est susceptible de donner comme de recevoir la forme), l'explosion de toute forme de l'autre ("plastic" et "plastiquage"). » Pour obtenir la pièce recherchée, il faut détruire le moule, il faut refondre le métal. Le sable comme le métal se retrouvent plus ou moins rapidement réintégrés dans un même cycle.

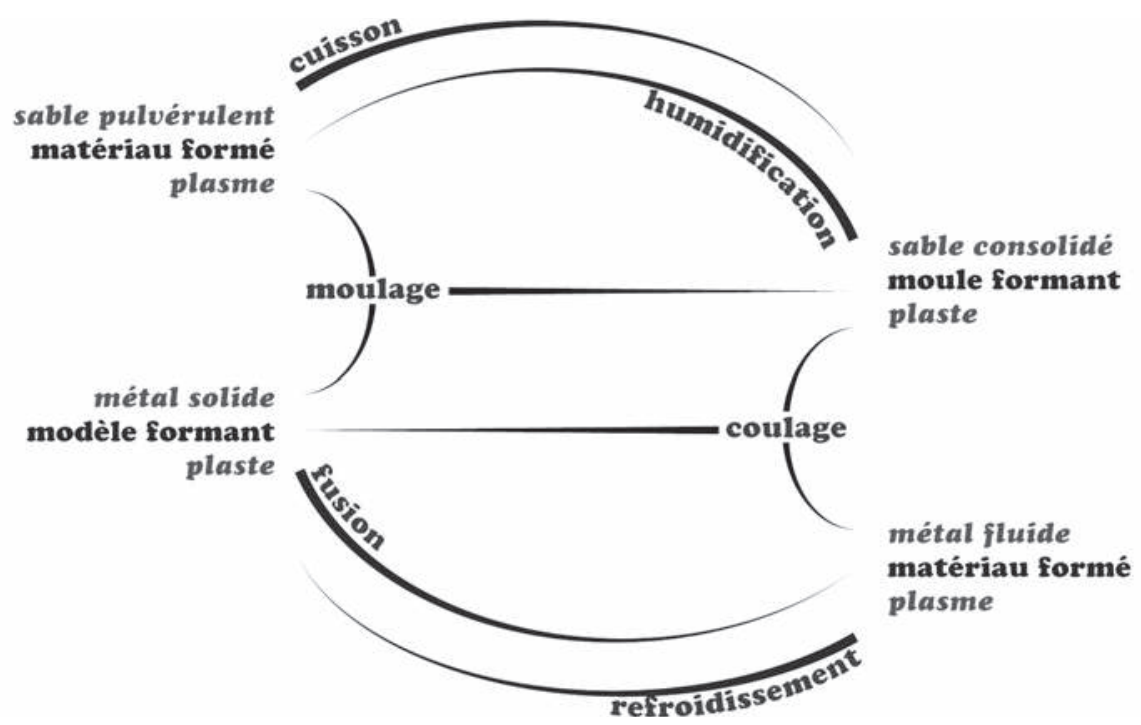

\section{Cycle plastique}

La technique de fonte au sable peut alors être envisagée comme un cycle plastique : le processus de prise/dation de forme par moulage/coulage apparaît comme étant le produit de la rencontre par pression de plasmes (êtres formés) et de plastes (êtres formants) constitués de matériaux passant d'un état plus ou moins fluide à un état plus ou moins solide par la modification de leur température, de leur humidité et de leur compacité. Appliquée au processus de fonte au sable, marqué par la cyclicité et comme composé de boucles de métamorphoses successives d'éléments contigus, eux-mêmes caractérisés par l'impermanence et la variabilité catégorielle, ce type de description épouse une conception peut être plus fidèle du processus de l'empreinte à l'œuvre dans maintes techniques de fabrication.

\section{$\&$}

Cependant c'est surtout la dimension cyclique de l'ontologie plastique développée en philosophie qui sied particulièrement bien à la logique technique observée à Fès où, pour reprendre le vocable d'Hannah Arendt (2005 [1961] : 144), le travail a pris le pas 
sur l'ouvrage. Aussi ce n'est pas par hasard si le terme « cycle » apparaît dès les premiers mots du film Pour Mémoire (La forge) que Jean-Daniel Pollet a consacré au déclin d’une fonderie industrielle française : «Un matin, un matin où rien ne commence. Simplement on entre dans un cycle. » À Fès, la cyclicité est aussi une conséquence des changements engendrés par l'industrialisation masquée des activités artisanales. Si la cyclicité n'est pas le propre de l'artisan œuvrant seul - pour lui le moulage ne constitue qu'une phase parmi d'autres (Benfoughal 1992) -, elle est en revanche celle du mouleur spécialisé qui ne confectionne plus de pièces unitaires mais des séries de pièces. Ne fabriquant pas lui-même les modèles, seule la préparation de la matrice lui permet de rompre un temps avec cette boucle perpétuelle. Car toutes les opérations qui précèdent et qui suivent sont un jeu d'empreintes répétitif (les deux fondeurs confectionnant chacun près de trois cents moules). La confection sérielle de moules est rendue possible par l'introduction de la matrice. Or cet élément est celui le plus délicat à confectionner : "Si tu ne sais pas faire ça, me disait un mouleur, autant ne pas travailler. » Sans la confection de cette matrice initiale, le travail de fonderie ne serait que la répétition d'opérations identiques et le mouleur condamné à l'exécution exclusive d'un travail cyclique très monotone. Outre l'expression de la logique technique à l'œuvre, la parfaite adéquation du processus observé avec la notion de cyclicité plastique témoigne de la bascule de pans entiers de l'artisanat marocain dans un univers ouvrier qui tait son nom. 


\section{NOTES}

Photo d'ouverture : La fonte au sable à Fès dans les années 1980 (Paccard 1981 : 460-463)

1. Aujourd'hui, l'usage de «sables » à prise chimique, ne contenant plus de véritable sable, a tendance à se généraliser.

2. Sur cette technique appliquée à l'aluminium et l'histoire de sa diffusion au sud du Sahara, voir Romainville (2009).

3. L'histoire de ces deux techniques, l'implantation ancienne de l'une, la diffusion récente de l'autre, est d'ailleurs confirmée par la sémantique locale : la première est dite beldi - terme qui désigne d'anciennes techniques locales -, tandis que la seconde est qualifiée de rumi - terme qui désigne des techniques récentes exogènes.

4. Concernant ces différents objets, voir successivement : Arminjon \& Bilimoff (2010 [1998] : 82), Remillet (2009), Benfoughal (1992), Romainville (2009) et Chochod (1909).

5. Aujourd'hui, la connaissance d'artefacts cuivreux (Souville 1986 : 105), la localisation avérée de nombreux gisements de cuivre (Rosenberger 1970), la mise au jour de quelques outils de mineurs (Jodin 1966 : 19) et l'existence de nombreuses gravures rupestres représentant des armes métalliques (Camps 1960) tendent à prouver que la zone géographique correspondant au Maroc actuel a connu un âge des métaux comparable à celui observé au nord de la Méditerranée.

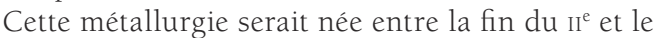
milieu du I $^{\mathrm{er}}$ millénaire, lorsque la civilisation d'El Agrar, après avoir diffusé ses techniques, a vu sa zone d'influence diminuer (Rodrigue 1999 : 132-133). C'est d'ailleurs de cette période, vers 1200 avant notre ère, que datent les premiers textes laissant penser que les « Berbères » connaissaient la métallurgie du bronze (Grébénart 1988 : 61).

6. Sur les pratiques métallurgiques de l'Orient musulman et de l'Andalousie, voir notamment Migeon (1927), Mayer (1959), Baer (1983) et Ward (1992).

7. Le terme tarziq encore employé pour désigner les châssis, définissant les limites extérieures des moules en sable, semble d'ailleurs avoir été directement associé au technolecte juif et confirme, si tant est qu'il faille encore le démontrer, leur importance dans les pratiques de la dinanderie : le dictionnaire de Marcelin Beaussier et Mohamed Ben Cheneb (2006 [1958] : 394) définit le terme par « châssis du ciseleur juif »; même si Alfred De Prémare (1995 : 106) n'a pas retenu cette définition, préférant celle, plus juste d'un point de vue technique, de « châssis du moule des fondeurs et des bijoutiers $»-$ Marcelin Beaussier et Mohamed Ben Cheneb emploient sûrement « ciseleur » comme terme générique pour désigner un artisan travaillant le métal, car le châssis n'est pas utilisé en ciselure.

8. Le décochage est l'opération qui consiste à séparer l'élément brut de fonderie de son moule. Il ne faut pas confondre le décochage avec le démoulage qui consiste, lors de la phase de préparation d'un moule, à retirer le modèle à reproduire du sable dans lequel vient d'être marquée son empreinte (Baudry \& Bozo $1978:$ 632).

9. La méthode de fonte au sable par empilement, spécialité plurimillénaire de la métallurgie chinoise (Arminjon \& Bilimoff 2010 [1998] : 87), permet aux dinandiers fondeurs de produire en grande quantité des pièces à partir de différents modèles sans noyaux ni entrants qui feraient obstacles au démoulage. Également appelé « coulée en presse » (Baudry \& Bozo 1978 : 632), ce procédé consiste « à utiliser plusieurs moules semblables en argile, superposés et assemblés, permettant de fondre plusieurs objets identiques en une seule coulée » (Arminjon \& Bilimoff 2010 [1998] : 86). Comme nous le verrons ci-après, afin de produire une série de moules semblables, les artisans commencent par confectionner une matrice, étape absente des processus de fonte au sable maghrébins décrits jusqu'à présent.

10. Sur le reste du processus et plus généralement cette activité et sa place dans la dinanderie de Fès, voir Buob (2009a : 255-279 et 375-379 et 2009b [visible en libre accès à l'adresse http://vimeo.com/76040806]).

11. Le sable jaune utilisé en fonderie contient généralement « de la silice pour être réfractaire sous l'action du métal liquide et de l'alumine pour avoir la consistance, la tenue ou le corps nécessaires. La teneur en silice oscille généralement entre 85 et $95 \%$ et celle en alumine entre 15 et $5 \%$. On y trouve parfois des oxydes de fer, de la chaux, etc. » (Gillot \& Lockert 1905 : 57).

12. À la fin des années 1990, dans l'Aurès algérien, les artisans fondeurs n'allaient plus eux-mêmes se procurer le sable jaune composé de grès altéré mais l'achetaient directement au marché. Ils continuaient cependant à confectionner eux-mêmes le mélange en ajoutant de l'huile d'olive comme liant afin d'obtenir une pâte 
grasse et humide (Benfoughal 1997 : 211). Toujours au Maghreb, les artisans kabyles employaient du blanc d'œuf comme liant (Camps-Fabrer 1970 : 50) là où des bijoutiers d'Asie centrale utilisaient du lait de chèvre (Soukharéva 1971, in Benfoughal 1997 : 211).

13. Le terme « matrice » est utilisé pour traduire le terme arabe «netwa » qui est littéralement un pluriel du mot «femelle» (Ferré 1955: 178). Dans le prolongement du « caractère obstétrique » de nombre de pratiques métallurgiques observées dans le monde (Eliade 1977 [1956] : 7), il serait tentant de voir ici une association métaphorique de la pratique de moulage à une forme de reproduction biologique - comme dans le cas de l'empreinte (Didi-Huberman 2008 : 53). Mais ce genre d'interprétation se ferait sans aucun doute au détriment de représentations locales dénuées de cette forme de symbolisme.

14. Action consistant à « produire une empreinte ou une partie d'empreinte en faisant pénétrer un modèle par pression dans le sable » (Baudry \& Bozo 1978 : 628).

15. L'opération de « serrage », aussi appelée « damage », consiste à « comprimer le sable contenu dans un châssis au moment de la confection d'un moule au sable sans toutefois supprimer la perméabilité qui est indispensable à l'évacuation des gaz » (Baudry \& Bozo 1978: 628).

16. Si André Paccard ne parle pas de "modèles », c'est pourtant bien ces éléments qu'il désigne. Il fait un usage impropre du terme « contre-moule » qui, en fonderie, désigne habituellement un moule qui en enveloppe un autre.
17. Selon Albert Gateau le terme « gabarit » pourrait même être issu du mot qâleb (1966 : 116).

18. Comme le rappelle Jacques Roux (2002 : 280), l'observation attentive du principe de moulage d'une brique par Gilbert Simondon (2005 [1964] : 45) a justement conduit à renverser cette conception : le matériau actif transporte le mouvement, là où la force statique de la forme le contraint.

19. Cette idée de la « relativité des catégories » appliquée aux corps plastiques est empruntée à André LeroiGourhan qui, en revanche, ne l'aborde que pour en minimiser la portée (2000 [1943] : 207).

\section{RÉEFERENCES}

Arendt, H. 2005 [1961] Condition de l'homme moderne. Paris : Calmann-Lévy.

Arharbi, R. 2003 L'Occupation du littoral du Maroc à l'époque préromaine. In Catherine Gaultier-Kurhan (dir.) Patrimoine culturel marocain. Paris : Maisonneuve et Larose : 57-101.

Arminjon, C., \& Bilimoff, M. 2010 (1998) L’Art du métal. Vocabulaire technique. Paris : Éditions du Patrimoine.

Baer, E. 1983 Metalworks in Medieval Islamic Art. Albany (États-Unis) : State University of New York Press.

Baudry, M.-Th., \& Bozo, D. 1984 (1978) La Sculpture. Méthode et vocabulaire. Paris : Inventaire des monuments et richesses artistiques de la France.

Beaussier, M., \& Ben Cheneb, M. 2006 (1958) Dictionnaire pratique Arabe-Français (arabe maghrébin). Paris : Ibis Press.

Benfoughal, T. 1997 Bijoux et bijoutiers de l'Aurès algérien. Paris : Éditions du CNRS.

— 1992 Chaîne opératoire et changement technique dans la bijouterie de l'Aurès, Algérie. In Hélène Balfet (dir.) Observer l'action technique. Paris : CNRS : 147-161.

Berque, J. 1962 (1939) Le Maghreb entre-deux-guerres. Paris : Éditions du Seuil. 
Buob, B. 2009b La Dinanderie de Fès, un artisanat traditionnel dans les temps modernes. Une anthropologie des techniques par le film et le texte. Paris: Ibis Press - Éditions de la MSH ; ouvrage contenant un DVD avec les quatre films de la série Hommes et objets de la dinanderie de Fès, visible en libre accès à l'adresse http://vimeo.com/album/2559829 [consulté le 19 novembre 2014].

Cambazard-Amahan, C. 1989 La Décoration sur bois dans l'architecture de Fès. Époques almoravide, almohade et début mérinide, Aix-en-Provence : Éditions du CNRS.

Camps, G. 1960 Les Traces d'un âge du bronze en Afrique du Nord, Revue africaine, 104 : 31-55.

Camps-Fabrer, H. 2013 [1992] Bijoux, Encyclopédie berbère, 10 ; http://encyclopedieberbere.revues. org/1758 consulté le 7 mai 2014.

— 1970 Les Bijoux de la grande Kabylie, Paris : Arts et Métiers Graphiques (Mémoires du Centre de Recherches Anthropologiques, Préhistoriques et Ethnographiques, XII).

Chochod, L. 1909 Note sur les procédés de fonderie employés en Annam, Bulletin de l'École française d'Extrême-Orient, 9 : 153-158.

Colin, G. S. 1936 Les Mines marocaines et les Marocains, Bulletin économique et social du Maroc, III, $13: 194-200$.

Combet, É. 2009 Du concept de plasticité à la plasticité du concept, Plastir, 14.

Delpy, A. 1958 Note sur un coffret, une lampe et un fragment de plateau du musée régional des arts et du folklore de Rabat, Hespéris, LXV : 165-167.

Didi-Huberman, G. 2008 La Ressemblance par contact. Archéologie, anachronisme et modernité de l'empreinte. Paris : Éditions de minuit.

El Koubtia, S. 1992 La Contribution des bijoutiers juifs à la bijouterie artisanale du Maroc du $\mathrm{x}^{\mathrm{e}}$ à la première moitié du $\mathrm{xx}^{e}$ siècle. Étude historique, socio-linguistique et symbolique. ». Université Paris VIII, département d'Études hébraïques (thèse de doctorat sous la direction de Haïm Zafrani).

Eliade, M. 1977 (1956) Forgerons et alchimistes. Paris : Flammarion.

Eudel, P. 1906 Dictionnaire des bijoux de l'Afrique du Nord. Paris : Ernest Leroux.

France, Cl. de. 2010 [1983] L'Analyse praxéologique. Composition, ordre et articulation d'un procès, Techniques \& Culture, 1 : 147-170.

Ferré, D. 1955 Lexique marocain-français. Fédala : Édition Nejma.

Gaube du Gers, O. 1993 Le Travail au tour à repousser, la fonte au sable, la fonte à cire perdue, Métiers d'art, $51: 36-37$.

Gateau, A. 1966 Atlas et glossaire nautique tunisien, vol. II. Beyrouth : Dar El-Machreq.

Gillot, A., \& Lockert, L. 1905 Nouveau manuel complet du fondeur de fer et de cuivre. Paris : Encyclopédie Roret.

Grébénart, D. 1994 Cuivre, Encyclopédie Berbère, 14 : 2153 -2157.

- 1988 Les Premiers métallurgistes en Afrique Occidentale. Paris : Errance.

Gsell, S. 1901 Les Monuments antiques de l'Algérie. Paris : A. Fontemoing.

Houssel, J.-P. 1966 L'Évolution récente de l'activité industrielle de Fès, Revue de géographie du Maroc, $9: 59-84$.

Ibn Abi Zar', A. 1990 (1326) Rawd al Qirtâs, traduit de l'arabe par A. Beaumier (1860). Rabat: Éditions La Porte.

Jodin, A. 1966 Les Gisements de cuivre du Maroc et l'archéologie des métaux, Bulletin d’archéologie marocaine, VI : 11-27.

Laborie, J.-P., \& Tag, B. 1990 Atlas de la médina de Fès. Toulouse : Presses universitaires du Mirail.

Lebon, É. 2012 Sable ou terre, in Élisabeth Lebon, Le fondeur et le sculpteur. Techniques du bronze et histoire de l'art. Paris : Ophrys - INHA (« Les Collections électroniques »), [en ligne] : http:// inha.revues.org/3468 consulté le 7 mai 2014.

Leroi-Gourhan, A. 2000 (1943) L'Homme et la matière. Paris : Albin Michel.

Magny, J. 1869 Histoire d'un morceau de verre. Paris : Brunet Éditeur.

Malabou, C. 2005 La Plasticité en souffrance, Sociétés \& Représentations, 20-2 : 31-39. 
— 2000 Le Vou de plasticité. In Catherine Malabou (dir.), Plasticité. Paris : Éditions Léo Scheer : 7-25.

Mayer, L. A. 1985 (1959) Islamic Metalworkers and Their Works. Genève : A. Kundig.

Migeon, G. 1927 Manuel d'art musulman, tome II. Paris : A. Picard.

Mordant, C., \& Pernot, M. (éd.) 1998 L'Atelier du bronzier en Europe du XX au viII siècle avant notre ère. Paris : CTHS.

Paccard, A. 1981 Le Maroc et l'artisanat traditionnel islamique dans l'architecture, tome II. Annecy : Édition Atelier 74

Prémare, A.-L. de. 1998 Dictionnaire Arabe-Français, tome X. Paris : L'Harmattan.

Remillet, G. 2009 Ethno-cinématographie du travail ouvrier. Essai d'anthropologie visuelle en milieu ouvrier, Paris : L'Harmattan.

Riché, R. 1961 La Corporation des bijoutiers à Constantine avant 1830, Revue africaine, 105 : 177-181.

Rodrigue, A. 1999 L'Art rupestre du Haut Atlas marocain. Des pasteurs du Dra aux métallurgistes de l'Atlas,. Paris : L'Harmattan.

Romainville, M. 2009 Les Routes africaines de l'aluminium, Techniques \& Culture 51 (1) : 74-97.

Rosenberger, B. 1970 Les Anciennes exploitations minières et les centres métallurgiques au Maroc. La vie des anciennes exploitations et la place des mines et des métaux dans l'histoire du Maroc, Revue de géographie du Maroc, 18 : 59-102.

Roux, J. 2002 Entre le moule et l'argile. La science des matériaux est-elle allagmatique ?, in Jacques Roux (dir.), Gilbert Simondon. Une pensée opérative, Saint-Étienne: Publications de l'université de SaintÉtienne : 274-298.

Rulhmann, A. 1935 Moules à bijoux d'origine musulmane, Hespéris, XXI : 141- 148.

Simondon, G. 2005 (1964) L’Individuation à la lumière des notions de forme et d'information. Grenoble : Éditions Jérôme Millon.

Souville, G. 1986 Témoignages sur l'âge du bronze au Maghreb occidental, Comptes rendus des séances de l’Académie des Inscriptions et Belles-Lettres, 130-1 : 97-114.

Terrasse, H., \& Hainaut, J. 1925 Les Arts décoratifs au Maroc. Paris : H. Laurens.

Ward, R. 1992 Islamic Metalwork. Londres : British Museum Press.

\section{Filmographie}

Buob, B. 2009a Fonderie, film documentaire, $78 \mathrm{~min}$, in La Dinanderie de Fès, un artisanat traditionnel dans les temps modernes. Une anthropologie des techniques par le film et le texte. Paris: Ibis Press - Éditions de la MSH ; visible en libre accès à l'adresse http://vimeo.com/76040806 [consulté le 19 novembre 2014]. Pollet, J.-D. 1978 Pour mémoire (La forge), film documentaire, 62 min.

\section{POUR CITER CET ARTICLE}

Buob, B. 2014 Sable, moules, modèles et matrice, in S. Boulay \& M.-L. Gélard, Vivre le sable! Corps, matière et sociétés, Techniques \& Culture 61: 122-143. 


\section{RÉSUMÉ}

Sable, moules, modèles et matrice. Le cycle plastique d'une fonderie marocaine. Il s'agit ici de reconsidérer des données concernant un procédé de fonte au sable filmé au Maroc il y a plus d'une dizaine d'années. Partant du double constat de la rareté des traces historiques laissées par cette technique et de sa relative rétivité à une analyse technologique classique en anthropologie filmique (en raison du constant changement d'état de ses deux principaux matériaux et de l'ambiguité fonctionnelle des éléments de sa composition), ce texte propose d'envisager la fonte au sable comme le produit de transformations éminemment plastiques. Permettant de se libérer en partie de catégories ambigües, telles qu'outil, objet et matière, la plasticité invite à penser le processus technique comme étant le fruit de rencontres successives entre des corps plasmes et des corps plastes, des «êtres formés » et des « êtres formants ». Le processus de fabrication n'est dès lors plus envisagé comme l'expression de la transformation d'une matière, à l'état naturel, en objet, à l'état fabriqué, mais comme un processus permanent de prise/dation/ destruction de formes. La réflexion en termes de plasticité se révèle particulièrement adaptée à la compréhension de la logique à l'œuvre durant ce processus marocain de fonte au sable, technique qui se caractérise par la cyclicité et l'instabilité catégorielle de ses composantes.

\section{ABSTRACT}

Sand, mould, pattern and matrix. Plastic cycle of a Moroccan foundry. The article reviews data on a process of sand casting that was filmed in Morocco more than ten years ago. It starts from the twin assertion that historical traces of sand casting are rare and that this technique does not lend itself easily to technological analysis which is commun in french visual anthropology (because of a constant change in state of its two principal materials and the functional ambiguity of some of its technical components). Sand casting is thus approached as the result of mainly plastic transformations. Since it allows to free oneself from ambiguous categories such as tool, object and matter, plasticity invites to consider the technical process as the product of successive encounters between "plasm" and "plast" bodies, between "formed beings" and "forming beings". The process of production is then no longer conceived as the transformation of a material in its natural state into a constructed object, but as a permanent process of taking/giving/destroying forms. Thinking in terms of plasticity is particularly suited to understand the logics operating in this moroccan process of sand casting, a technique characterized by the cyclicality and categorial instability of its components.

\section{MOTS CLÉS}

Dinanderie, Maroc, fonte au sable, moulage, cycle plastique

\section{KEYWORDS}

Brassware, Morocco, sand casting, moulding, plastic cycle 